\title{
Contribución al Estudio de Temperatura Basal
}

\author{
Por el Doctor Ricarion Rueda \\ Interno Rotatorio del Hospital San José de Bogotá
}

Fue por primera ves Rabutacan (1) en el ano 1780 quien senalo la lla mada febricula premenstual. Posteriormente Van Velde (1) fue of primero en Wacionar este fenomeno con el funcionamento del gonado femenino, obses. ando que el hombre la nina prepuber y la mujer en menopiasia natural o artificial no presentaban varaciones en su temperatura.

Mas tarde Palmer y De Villers citades por Ahumada (1), hicieron el st guicente experiments

Invectaron estradiol y progesteroma a mujeres que habian side sometid. a castracion por algun procese patologion de los oxarion l por consiguicute con curva termica uniforme, obersando una baja de la comperatura cuando apli

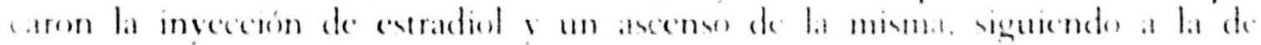
irogesterona.

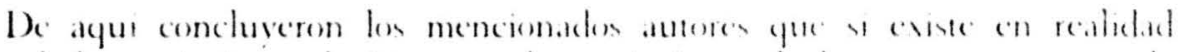
de rerdad una intima relacion entre las vartaciones de la rempetatuma tomada en condiciones basales y les homones ovarioss y por consigurente con la fisio logita del gonado temenino.

Posteriomente infinidad de autores entre lex que se cuenta especialmente Rubestain ("). han estudiado el fonomeno concturender que as un tent de gran

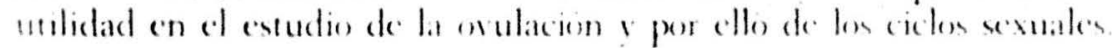

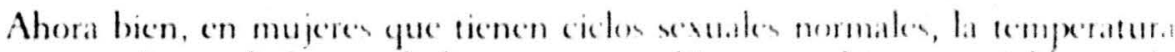

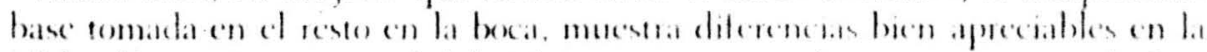

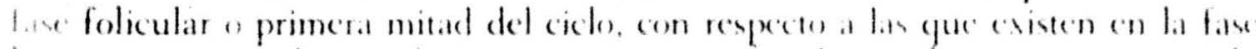
fluteinica o segunda mitad, fenomeno que concuerda con las eyperiencias reali udua por Palmer y De Villers. 
Durante la fase estrogénica la temperatura tomada en condiciones basales se sostiene por debajo de $\operatorname{los} 37^{\circ} \mathrm{C}$. para alcanzar y aún sobrepasar esta cifra en la fase luteínica, existiendo diferencias que oscilan entre 3 y 5 décimas de urado; la temperatura se sostiene entonces por encima de los $37^{\circ} \mathrm{C}$. hasta la siguiente menstruación, epoca en que vuelve a cifras inferiores semejantes a las de la fase folicular.

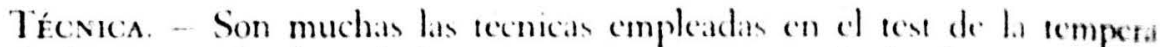
tura de base, y todas han dade segun sus autores, resultados bastante fieles

Kleitman ( ${ }^{3}$ ) de Chicago, acostumbra toman lat temperatura en las hotas de la tarde, y solamente advierte a sus pacientes que guarden una hora de re poso en cama, y que se abstengan de tomar bebidas calientes por media hora iv frías por una hora, antes de que la temperatura sea tomada.

La mayoría de los que se ecupan de ésto, aconsejan tomarla en la mañana al despertarse, pues dicen que las condiciones de reposo del cuerpo son indu. dablemente mejores, ya que el tiempo destinado a éste ha sido más prolongado.

Finalmente, algunos otros ginecologos aseguran que la prueba resulta ma fidedigna si se toma la temperatura en las horas de la manana al despertarse luégo a la tarde, claro está, despues de llenar las condiciones antes anotadas, o decir, una hora de reposo en cama y abstencion de alimentación liquida y solida por el mismo tiempo.

Ahora bien, como la temperatura empieza su ascenso o descenso a cualquier hora del dia o de la noche, el ideal para siber en qué momento se produce el cambio, sería tomar la temperatura con intervalos de una hora, cosa que sera laboriosa e incomoda para la paciente, además de carecer de valor práctico y que, para que el mencionado cambio enga veracidad necesita sosienerse pot algunos dias. Por cllo conclúmos que para lidelidad del test, son mas que acep tables cualquiera de las tree tecnicas mencionadas al principio.

La técnica que empleamos en el Departamento de Esterilidad del Hosputal San José, de Bogotá. Es una recopilacion hecha por el doctor Rodulfo Camezo (4), de las que se siguen en el llospital Rivadavia de Buenos Aires (Servicio def doctor Guillermo Di Paola), de la consulta de esterilidad del New York Hospi tal y del Margares Sanger Research de la misma ciudad.

En primer lugar se da instrucción impresa a la paciente en la forma que expreso a continuacion:

a) Debe anotar en una libreta o cuadernillo únicamente destinado á ests efecto la temperatura marcada por el termómetro.

b) Usar el termómetro centigrado en la boca.

c) Tomar la temperatura inmediatamente después de despertar y antes dh emprender cualquier actividad. 
d) Dejar el termómetro al lado de la cama la noche anterior y ascgurarse yue ha quedado listo para marcar apropiadamente, es decir que la columna de mercurio este por debajo siquicra de los $35^{\circ} \mathrm{C}$.

e) El tremometro debera permanecer en la boca por un tiempo no menor 15 minutos

f) Luego de kédo el dato suministrado por el termometro, apuntese en la libreta o cuadernillo frente a la fecha del dia correspondiente

g) Si algún incidente ha ocurrido durante la noche o en la mañana antes de la toma de temperatura, debe anotarse frentc a ésta usando los siguientes signos convencionales:

l-Insomnio

O-Relaciones sexuales.

D-Dolor pélvico intermenstrual.

F-Flujo.

H-Hemorragias intermenstruales.

Y-Ingestión de bebidas alcohólicas.

R-Resfriado.

E-Cualquier otra enfermedad.

h) Finalmente con una $\mathrm{X}$ los dias de la menstruación también frente al dato de la temperatura.

Ejemplo:

$$
\begin{array}{lrrrr}
\text { Julio } & 9 & 53 & 36,3 & X \\
\text { Julio } & 10 & 53 & 36,2 & X \\
\text { Julio } & 11 & 53 & 36,5 & F \\
\text { Julio } & 12 & 53 & 36,4 & 0 \\
\text { Julio } & 13 & 53 & 36,5 & 1
\end{array}
$$

Esta libreta es llevada por la paciente semanalmente a la consulta de les wrilidady los datos presentes en ella son pasados al cuadro (Modele original yate se incluve a continuación. Fig. No 1.

Sentadas ya estas bases generales entramos ahora a analizar los tipos de curvas que nos pueden suministrar datos de valor.

La grafica ideal, que nosotros hemos encontrade en mus pecas acisiones pro que autores como Fingle (") cuentit con numerosos cases, ce lit siguiente 1.g. No2).

Desperes de la última menstruacion la temperatura se sosticne por debare

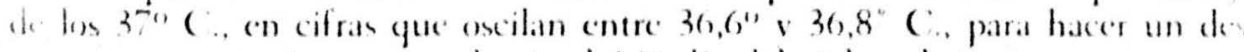
conse pequeno mas o menos hacia el 14" dia del ciche y luego un asecense que pasat de los $37^{\circ} \mathrm{C}$. que se sostiene con muy pocas variaciones hasta la siguient: menstruación. Esto claro está, en mujeres con ciclos de 28 dias. 
Este descenso, seguido de ascenso de la temperatura es considerado como el momento en que se hace la ovulacion, $y$ autores de la experiencia de Tomkins, de Filadelfia ("). diecn que posiblemente la caida previa de la curva termica se nala la osulacion ya que ha observado graficas indicadoras que el coite fecun dante se verifico en el dia de la carda.

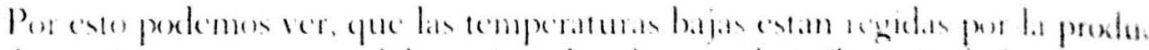

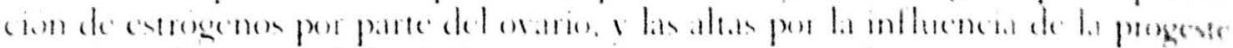

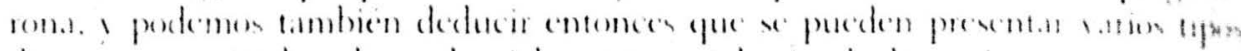

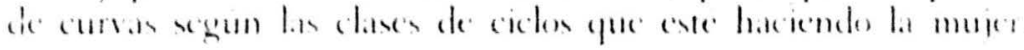

En un cicho bifisico, la cursa sera con variaciones mus pequenas de la que anotamos anteriormente, es decir temperaturas por debajo de 370 ( $\therefore$ durante lo primeros It dias, y por encima durante los vilumes it.

En un cicle trifásico, en que persiste la hormona del cuerpo amarillo u hormona progestacional y en que la hipofisis hace sentir su accion medante ia produccion del luteotropina, es logico que la temperatura se mantenga en cifras por encima de los $3^{-0}$ C. durante todo el cmbaram para deseender en minado este, y de ayu que algunos como de Protesor Aparicio (5) denominen al eent de la cemperatura basal el "Friedman de los pobres"

I a propesite del estudio de la temperatura de base en el combaraze, exise

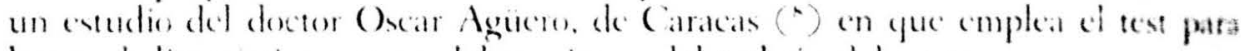
hacer el diagnostico precose del comienen del trabajo del parro.

1) dector Aguero llama la atencion sobre el descense de la curva termas a

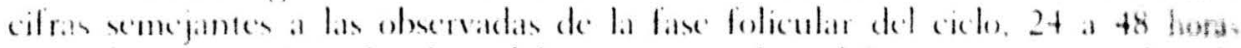

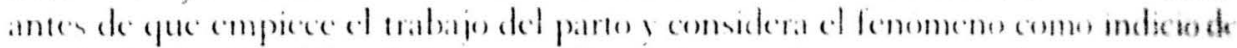

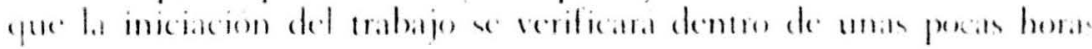

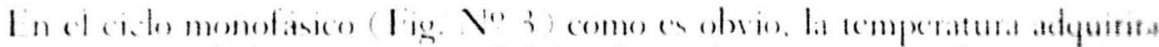

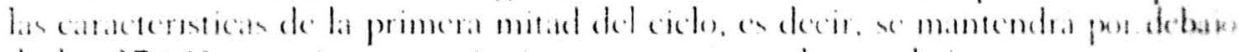

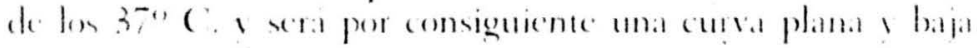

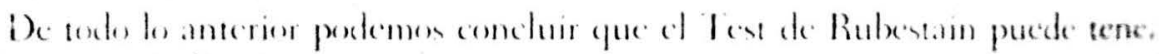
las igutientes indicationes

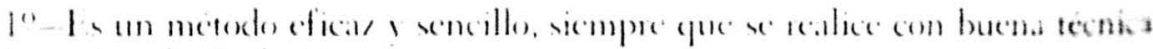
para investig.tr lis oxulacion.

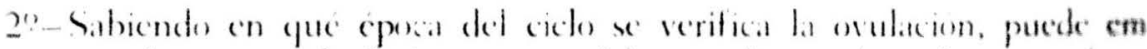
plearse para deserminar la fecha en que deben realizare las relaciones sexus

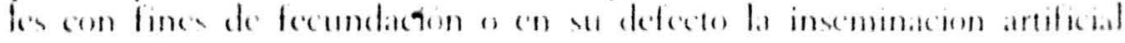

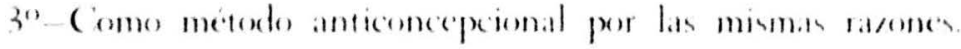

4"-Mediante el cest se puede hacer un diagnestico precos de embaras y determinar más a menos cuando se va a iniciar el trabajo ded parto. 
TEMPERATURA BASAL
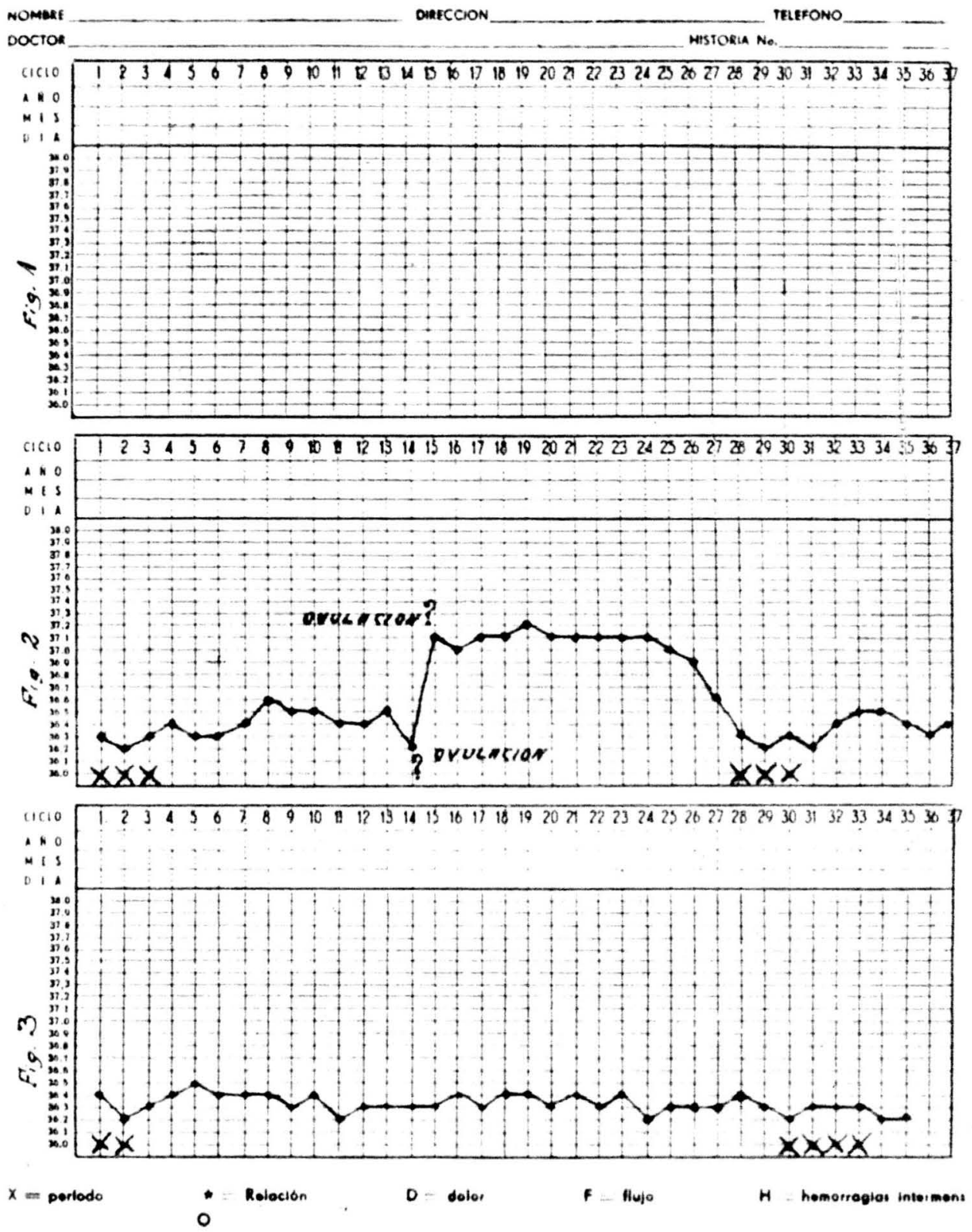
50- Vos sirve tambien como auxiliar en el diagnostico de los ciclos mo nolinicos.

60-Finalmente para-determinar la bondad de un tratamiento hormonal

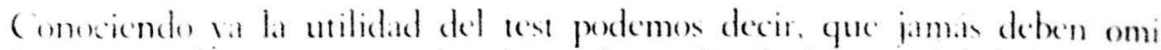

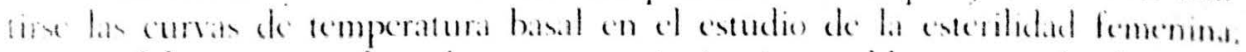

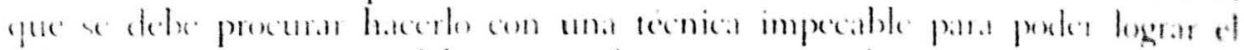

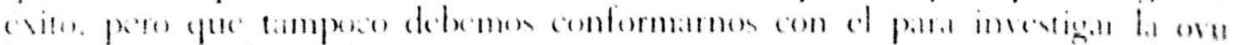

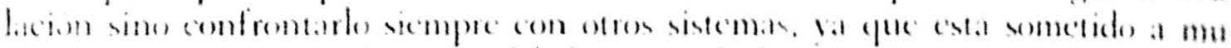

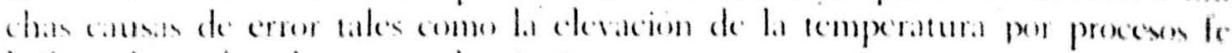
brike el compleo de ma mala reanical, ete.

\section{REFERENCIAS :}

1. Ahumada, Juan C.: "Tratado Elemental de Ginecologia". III Edicion. Tomo 1. Píf. 147. Lopez y Etchegoyen. Buenos Aires, 1952.

2. Crossen, H. S. y Crossen, R. J.: "Enfermedades de la Mujer". II Edición. Tomo 1. làg 101. Union Tiposrafica, Editorial Hispano Americana. Mexico, 1949

3. Cireenhill, J. P.: "Use of basal temperature graphs in determining date of ova. lition". J. A. M. A., 124; 698: 700. Mar. 1944. Cita del Year Book of Obstetrio and Gynecology. Pag. 336. The Year Book Publishers. Chicago, 1944

4. Camero C., Kodulfo: "Referencia personal y cuadros modelos de varios hospi, tale...

5-f. Engle, Earl T.: "Diagnostico y Tratamiento de la Esterilidad Humana" Primera edicion Jags. 132-159. "Beta", Buenos Aires, 1948

7.-Aparicio J., Arturo: "Conferencias de Ginecologia dictatas en el Hespetal Sart Juan de Dion de Bogoti, 1952.

8. Aguero, Oscar: "Temperatura Basal y conienzo del Trabajo de Parto". Boletin de la Matcrnidad "Concepcion Palacios". 3: 7, 91 a 95. Juho, 1952 Caracas. Venczuela 